\title{
The Full Plate: Benefits of simulated and raw data digital patterns in the Powder Diffraction File $^{\text {TM }}$
}

\author{
S. Gates-Rector, V. Bosnic, S. Kabekkodu, J. Blanton and T. Blanton \\ International Centre for Diffraction Data, Newtown Square, Pennsylvania, USA. \\ gates-rector@icdd.com
}

The Powder Diffraction File ${ }^{\mathrm{TM}}(\mathrm{PDF} \circledast)$ is a comprehensive materials database containing data for inorganic materials including minerals (natural and synthetic), metals and alloys, and high-tech ceramics, as well as organic materials such as pharmaceuticals, excipients and polymers. Databases, including the PDF, that provide structural details can be used for a range of materials characterization analyses, including (but not limited to) phase identification, quantitative analysis, and structure modelling for Rietveld refinement and whole-pattern fitting. As a result, structural databases are one of the key tools used in the crystallographic community [1]. Though these databases do tend to have some common applications, they often differ in content, format, and functionality. ICDD's PDF databases primary purpose is to serve as a quality reference tool for the powder diffraction community.

Historically, the PDF has contained entries constructed as d-spacing and intensity (d-I) reduced diffraction pattern representations for phase identification. These condensed entries reduced storage space requirements, and increased search speed capabilities. With the advancement of computer hardware and software, and the transition of the PDF to a relational database format, storage space and speed capabilities have become less limiting [2]. Over time the PDF has grown exponentially and has evolved to where it is now common practice to construct entries of full digital patterns. In addition to being a powerful characterization database used for the analysis of single and multi-phase X-ray diffraction data, the ICDD has systematically been adding raw data digital pattern references for crystalline and non-crystalline materials since 2008; with an emphasis on excipients and polymers [3]. The addition of full digital patterns has enabled the analysis and identification of disordered and amorphous materials using a combination of the raw data pattern and d-I lists, or whole pattern similarity searching. The evolution of raw data archiving in the Powder Diffraction File will be discussed in this presentation, with emphasis on the benefits and increased capabilities for characterization of materials in both research and industrial applications including pharmaceutical, forensic, and energy sectors.

[1] Kuzel, R. and Danis, S. (2007). Materials Structure. 14, pp.89-96.

[2] Gates-Rector, S., \& Blanton, T. (2019). Powder Diffraction, 34(4), pp. 352-360.

[3] Fawcett, T., Gates-Rector, S., Gindhart, A., Rost, M., Kabekkodu, S., Blanton, J., \& Blanton, T. (2019). Powder Diffraction, 34(2), pp. 164-183.

Keywords: database; raw data; PXRD; powder diffraction 